\title{
Electromagnetic coupling in a planar periodic configuration of resonators
}

\author{
C. Jouvaud ${ }^{* 1,2}$, A. Ourir ${ }^{2}$, and J. de Rosny ${ }^{2}$ \\ ${ }^{1}$ CEA, DAM, Gramat, F-46500 Gramat, France \\ ${ }^{2}$ Institut Langevin, Université Denis Diderot Paris 7, UMRS CNRS 7587, ESPCI, \\ 10 rue Vauquelin 75231 Paris cedex 05, France. \\ *camille.jouvaud@espci.fr
}

\begin{abstract}
We study theoretically small arrays made of a periodic arrangement of sub-wavelength resonators. Each resonator is modeled as an electrical dipole and a magnetic dipole. The two dipole moments are driven by the same complex amplitude. We show that the relative strength of the two dipoles strongly depends on cell symmetry. First, under this approximation, the dispersion relation is extracted for an infinite size array. Second, the diagonalization of the mutual impedance between cells matrix provides a powerful way to deduce the resonant frequencies for a finite size array. These results are validated numerically. To that end, we simulate two arrays of 4 by 4 multigap split ring resonators. Finally, an experimental demonstration of a tunable antenna based on this study is presented.
\end{abstract}

\section{Introduction}

Behavior of electromagnetic waves in periodic medium in which the lattice is much smaller than the wavelength is a challenging field. Metamaterials are composite structures, which involve periodic arrays of sub wavelength inclusions. By designing the elementary cell and controlling some parameters like the permittivity and/or the permeability, metamaterials can make up global mediums with interesting properties, like a negative refractive index.[1]

Most of the time, resonant cells of a metamaterial are designed to minimize the coupling between cells Consequently, the metamaterial resonance is at same frequency that the one of the unitary cell. This condition is required to apply theory of homogenization. Nevertheless, coupling effect always occurs and its preponderance is only a question of relative strength with the self-resonance of the cell.

The coupling in an array of half wavelength long rods that are only spaced by less a tenth of a wavelength have been studied in [2]. It has been shown that the coupling induces a strong splitting of the resonance frequencies. This phenomenon is analogous to Kronig-Penney potential wells in solid state physics [3].

In this paper, we investigate the behavior of a finite array of split ring resonators (SRR) popularized by Pendry et al.[4].
We develop a magneto-electric dipolar theory to model the coupling effect between the resonators. Finally, we study the far-field radiation of the finite structure.

Two types of array are studied. Both of them consist of 4 by 4 multigap split ring resonators. For the first one, we use an asymmetric square SRR with 3 gaps at the center of 3 sides. For the second one, we use a symmetric square SRR with 4 gaps at the center of the 4 sides. In Fig.1, a small loop placed above one SRR excites the 3-gap array. We clearly observe the splitting of the main resonance. This splitting results from the coupling effect between the resonators. When the small loop antenna is placed above the second structure, the splitting phenomenon remains but fewer resonances are observed (Fig.2).

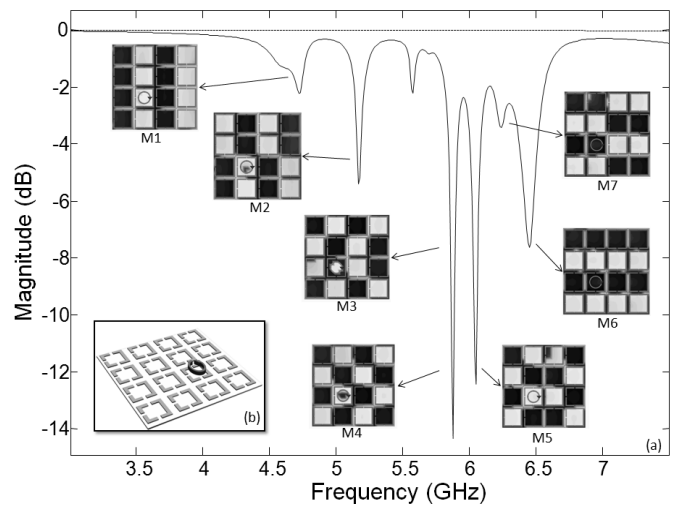

Figure 1: (a) Reflection coefficient of a small loop antenna. The M1 to M7 gray level maps show the normal magnetic field at 7 resonant frequencies. (b) the 4 by 4 array of $3-$ gaps SRR. In red, the small loop antenna.

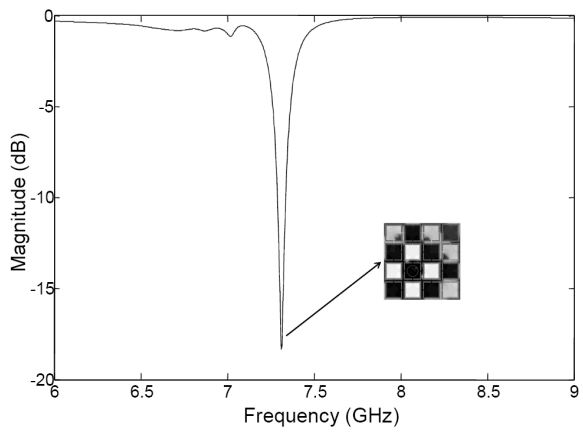

Figure 2 : Reflection coefficient of a small loop antenna of a 4 by 4 array of 4 -gaps SRR 


\section{A dipolar model}

We propose an original coupling model which is a generalization of the work published in [5][6]. Close the resonance frequency of an individual cell, the mode complex amplitude (e.g., the complex current) is the only degree of freedom of the cell. Moreover we assume that the current and charge distribution associated to the fundamental mode induces only an electrical and a magnetic dipole. Same approximation has been proposed with a Lagrangian approach in [7].The expression of the dipoles moments are given by the geometry of the elementary cell:

$$
\mathbf{P}=\frac{I d \mathbf{u}_{d}}{i \omega}, \quad \mathbf{M}=I S \mathbf{u}_{m},
$$

where $d$ and $S$ are the characteristic length and an effective surface of the electric and magnetic dipoles, respectively.
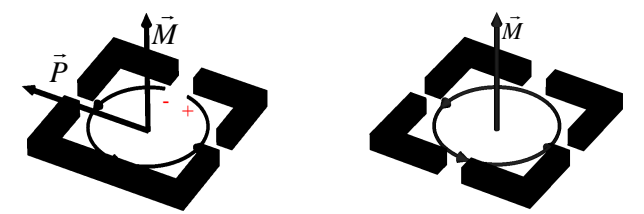

Figure 3: The dipolar model for a 3-gap and an 4-gap SRR.

Due to symmetry, the fundamental mode of a 4-gap SRR only generates a magnetic dipole. Indeed the structure geometry shows 3 symmetry planes: one horizontal and two vertical. Because the current and charge distribution are symmetric with respect to the horizontal plane but antisymmetric with respect the vertical ones, the electrical moment is null and the magnetic moment is vertical. Because, for the 3-gap SRR, one symmetry plane is broken, an electric dipoles disposed as shown in Fig.3 can appear. In other words, the electric dipoles introduced by the 4 gaps cancel by pairs for the full symmetric cell, which is not the case for the 3-gap cell.

Near-field radiations of the electric and magnetic dipoles at a distance $r$ are given in Eq.2.

$$
\begin{aligned}
& \mathbf{E}=\frac{1}{4 \pi \varepsilon_{0}} \frac{\left(3\left(\mathbf{u}_{d} \cdot \mathbf{P}\right) \mathbf{u}_{d}-\mathbf{P}\right)}{r^{3}} \\
& \mathbf{B}=\frac{\mu_{0}}{4 \pi} \frac{\left(3\left(\mathbf{u}_{m} \cdot \mathbf{M}\right) \mathbf{u}_{m}-\mathbf{M}\right)}{r^{3}}
\end{aligned}
$$

With this approximation, an analytic expression of the coupling between two cells can be obtained. We choose to describe the coupling in terms of electrical impedance, i.e., the FEM induced on a cell when a current flows through another one.

$$
e m f=-i \omega \iint \mathbf{B}_{1} \cdot d \mathbf{S}_{2}+\oint \mathbf{E}_{1} \cdot d \mathbf{l}_{2}
$$

The mutual coupling impedance describes the interactions between two cells. As for the self-impedance matrix $\mathbf{Z}_{\mathrm{s}}$, it models the interaction of a cell on itself. Because, we deal with simple resonators, we assume that the electrical behavior of each resonator is well described by a RLC (resistance, inductance, capacitive) resonator.

\section{Currents determination}

The complex amplitudes of the intensity currents on each cell are deduced from the Kirchhoff's voltage law applied on each cell. A set of $N$ equations, where $N$ is the number of cell is obtained:

$$
\left(i \omega L_{s}+\frac{1}{i \omega C_{s}}+R_{s}\right) I(l, m)+\sum_{l^{\prime \neq l}, m^{\prime} \neq m} Z_{s}\left(l^{\prime}-l, m^{\prime}-m\right) I\left(l^{\prime}, m^{\prime}\right)=0
$$

where $l$ and $m$ give the column and raw number of the cell. The dispersion relation is deduced from (4) when $N$ is very large. Limiting the coupling to the closest neighbors, an explicit and simple dispersion relation is worked out for a 3gap (see Fig.4(a)) and 4-gap arrays (see Fig.4(b)). From this relation, we can derive the span of the frequency splitting of the fundamental resonance. Those two curves reflect the magnetic and electric coupling for the first structure and the magnetic coupling for a second one.

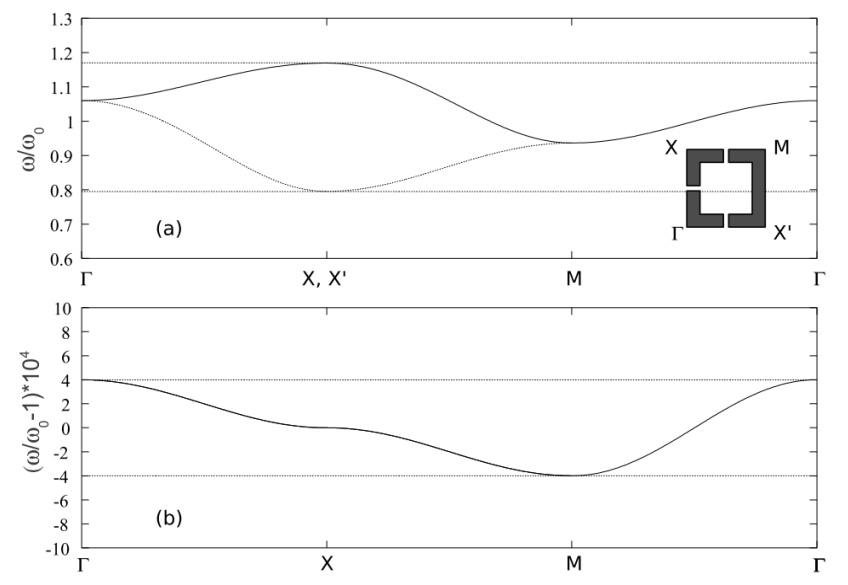

Figure 4: Dispersion relations for two arrays of 3-gap cells (a) and of 4-gap cells (b). The dispersion relation is plotted on the 4 major axis of the Brillouin zone. The dashed lines show the minimum and maximum values.

The slope of the dispersion relation of the array made of 4-gap cells is negative (backward waves) because the magnetic coupling between two parallel loops is negative. The slope sign of the 3-gap dispersion relation is either positive or negative. Along the $\Gamma X$ axis, the wave is vector is parallel to the electrical dipoles. The dispersion curve is therefore positive because the coupling of aligned dipoles is positive. Along the $\Gamma X^{\prime}$ axis, the wave vector is perpendicular and the coupling negative.

For finite size system, the complex amplitude on each resonator is deduced from the inversion of the sum of the self $\mathbf{Z}_{\mathrm{s}}$ and mutual impedance matrices $\mathbf{Z}_{\mathrm{m}}$. The self impedance matrix is proportional to the eye matrix times $i \omega L_{s}+1 / i \omega C_{s}+R_{s}$. The mutual impedance matrix is a $\mathrm{N}$ by $\mathrm{N}$ matrix with 0 on the diagonal elements. Thanks to these two matrices, Eq (4) can be rewritten in a concise way: 


$$
\mathbf{I}=\left(\mathbf{Z}_{m}+\mathbf{Z}_{s}\right)^{-1} \mathbf{S}
$$

where $\mathbf{S}$ and I are two vectors of $N$ elements that contains the FEM induced by the source and the complex current intensity on each cell.

We show that the resonances occurs when the imaginary part of the eigen-values of $\mathbf{Z}_{\mathrm{m}}+\mathbf{Z}_{\mathrm{s}}$ are equal to zero. Because $\mathbf{Z}_{\mathrm{s}}$ is proportional to the eye matrix a resonance appears when the imaginary part of the self impedance is opposite in sign to the eigenvalues of $Z_{\mathrm{m}}$. For 4-gap structure, only one main resonance is observed because coupling effects are too weak to induce a mode splitting effect. This is due to the fact that the $\mathrm{Q}$ factor is too small compare to resolve the different modes. In other words, all modes are degenerated.

Fig. 5 shows the calculated resonant modes for the 3 gaps structure. As expected, we observe that frequency position and the amplitude of the modes depend on both the mutual and the self impedance of the resonators. The 3-gap SRR array is dominated by the electrical dipole coupling. This result is in complete accordance with [8].

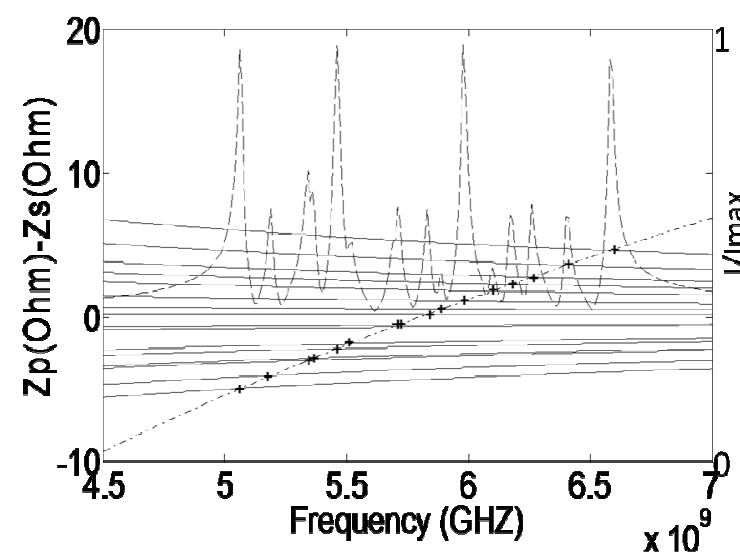

Figure 5: Frequency dependence of : the self impedance versus frequency (dash-dotted line), the imaginary part of the eigen-values of the mutual impedance times minus one (continuous line) and the current of one particular cell (dashed line). These results are obtained with an array of 4 by 43 -gap cells.

\section{A dynamic antenna tuning and beamforming}

The eigen-modes of finite size arrays are poor radiators because the spatial oscillations of the mode patterns are smaller than a wavelength and sub-wavelength modes hardly couple to propagative waves. However the Purcell's effect can mitigate this limitation. Indeed, the strong resonance effect significantly enhances the radiated power and compensates for the impedance mismatch.

The studied structure can thereby be used as an antenna emitting at these frequencies. With formalism presented above, the antenna pattern is simply worked out from the current distribution of the array. On Fig. 6, instead of tuning the fundamental frequency of the single cells, we electrically tune the resonance frequency of the cells thanks to semiconductor diodes which have a variable capacitance that is a function of the D.C. voltage applied on its terminals (varicaps). Fig. 7 shows the pattern of the radiated field for different D.C. voltages. The different patterns correspond to different eigen-modes.

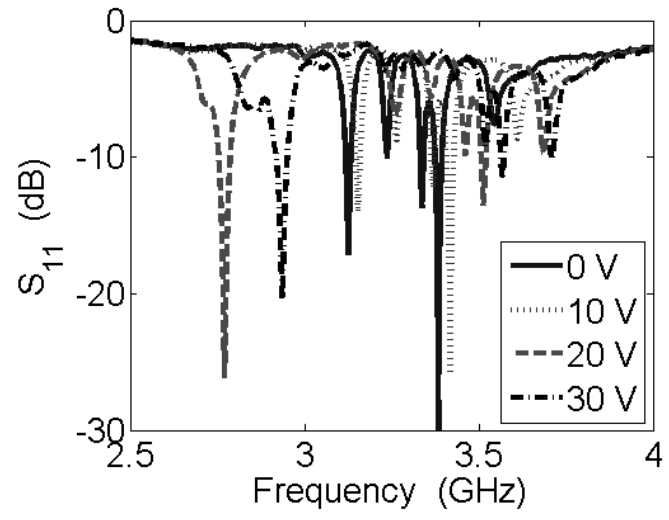

Figure 6: Measured reflection coefficient of a small loop antenna that is above a 4 by 4 array made of 3gap cells for different voltage applied on the varicap (see text).

Thus, a strongly tunable directive antenna can be realized by adjusting the resonance frequency. The advantage of this antenna is to show a small form factor, a tunable radiation directivity and a good matching. The far field polarization of this antenna is vertical, i.e., the polarization is linear and electric field is polarized in the plane substrate. Now, the goal of the future works is to adjust individually the resonance frequency on each cell in order to obtained a controlled directivity pattern.
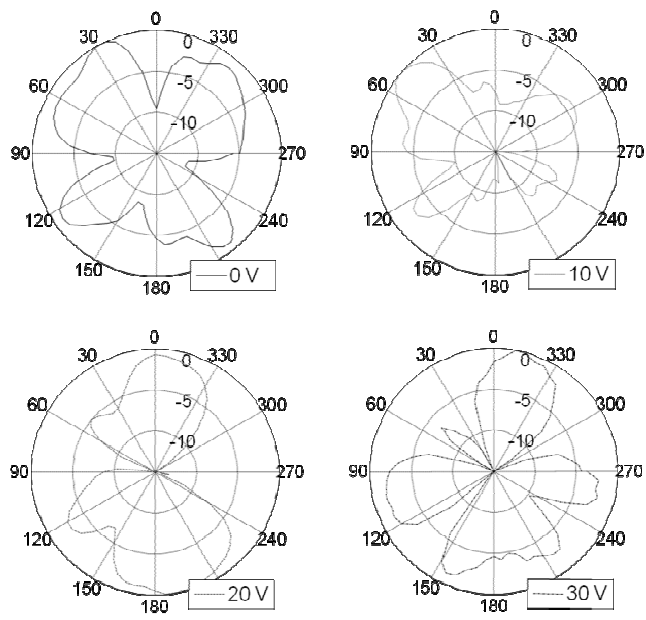

Figure 7: Measured radiation pattern of a small array of 3 gaps SRR for different applied voltage on the Varicaps for the same frequency.

\section{Conclusions}

We have developed a model based on impedance formalism for infinite and finite size array made of resonant cell. An electric and a magnetic dipole associated to each cell model 
the coupling between the fundamental modes of the resonators. Based on this approach, the electromagnetic behavior of such a structure is explained. The electromagnetic coupling induces a frequency splitting of the fundamental mode of a single cell. The dispersion relation leads to an estimate frequency span of the modes. Diagonalization of the mutual impedance matrix explains why the fundamental mode is splitted into $N$ modes where $N$ is the number of cells of the array. Finally an application to an electronically tunable directive antenna is proposed. This small antenna shows good radiation efficiency and tunable bandwidth.

\section{Acknowledgments}

This work has been partially supported by the French National Agency (ANR) for its financial support with the project OPTRANS (number 2010 BLAN 0124 04). We gratefully acknowledge the financial support of the French government-funded technological research organization CEA/DAM.

\section{References}

[1] D. R. Smith et al. " Composite Medium with Simultaneously Negative Permeability and Permittivity", Phys. Rev. Lett. 84 , 4184 (2000).

[2] Lemoult F., Lerosey,G., Rosny, J., and Fink, M., "Resonant Metalenses for Breaking the Diffraction Barrier", Phys. Rev. Lett. No.104, 20390,1 (2010).

[3] R. Kronig and W. G. Penney, Quantum mechanics of electrons in crystal lattices. Proc. R. Soc. London Ser. A, 130,499-512 (1931).

[4] J. B. Pendry, A. J. Holden, D. J. Robbins, and W. J. Stewart, "Magnetism from conductors and enhanced nonlinear phenomena", IEEE Trans. Microwave Theory Tech. 47, 2075 (1999).

[5] Marques, M., and al,"Role of bianisotropy in negative permeability and left-handed metamaterials" , Phys Rev B. No. $65,144440,(2002)$.

[6] E. Shamonina, V. A. Kalinin, K. H. Ringhofer, and L. Solymar, "Magnetoinductive waves in one, two, and three dimensions" J. Appl. Phys. 92, 6252, (2002).

[7] Sersic, I.; Frimmer, M.; Verhagen, E. \& Koenderink, A. F."Electric and Magnetic Dipole Coupling in NearInfrared Split-Ring Metamaterial Arrays" Physical Review Letters, 103, 213902, (2009).

[8] Katsarakis, N.; Koschny, T.; Kafesaki, M.; Economou, E.N \& Soukoulis,C.M."Electric coupling to the magnetic resonance of split ring resonators"Applied Physics Letters, AIP, 84, 2943-2945, (2004). 